\title{
HIV and Periodontal Disease: Redemption or Resurrection
}

\author{
Ashita .S. Uppoor ${ }^{1 *}$ and Dilip .G. Nayak ${ }^{2}$
}

${ }^{1}$ Department of Periodontics, Manipal College of Dental Sciences, Mangalore, Karnataka, India ${ }^{2}$ Professor \& Associate Dean, Manipal College of Dental Sciences, Mangalore, Karnataka, India

\begin{abstract}
Human immunodeficiency virus (HIV) infection, a major medical crisis that has hit families, communities \& nations across the world, remains a major health concern of unprecedented dimension. Mounting evidence suggests that co-infections and inflammation may result in re-activation of latent virus and blunt the success of Highly Active Antiretroviral Therapy (HAART). Periodontal diseases are chronic multibacterial infections that can result in significant bacterial \& inflammatory load in the body and HIV infection has been found to be associated with various forms of periodontal diseases. Besides Immune Reconstitution Inflammatory Syndrome (IRIS), following HAART, may also lead to increased frequency of periodontal disease among these patients. If co-infections \& inflammation can increase the risk of HIV reactivation, can periodontitis in an HIV infected individual lead to HIV re-activation. This question remains unanswered, but initial evidence suggests a probable role of periodontitis in HIV re-activation. The clinical implication of such a role is that, periodontitis being most often preventable and definitely treatable, every attempt should be made to prevent and promptly treat this disease in individuals with HIV infection.
\end{abstract}

Keywords: Periodontal disease; HIV infection; Inflammation; Reactivation

\section{Introduction}

History of HIV (human immunodeficiency virus) and AIDS (acquired immunodeficiency syndrome), dates back to 1981 when Centre for Disease Control reported five cases of pneumocystis carinii pneumonia in homosexual men in California. A major medical crisis that has hit families, communities and nations across the world, AIDS has already caused the death of over 30 million people worldwide. Several reports have identified a strong correlation between HIV infection and various oral lesions like oral candidiasis, hairy leukoplakia, Kaposi's sarcoma, non Hodgkins lymphoma \& specific forms of periodontal disease [1]. Periodontal diseases that are most strongly associated with HIV infection include linear gingival erythema, necrotizing ulcerative gingivitis and necrotizing ulcerative periodontitis [2]. Of current interest are the proposed mechanisms by which periodontitis and the pathogens involved may cause reactivation of HIV and the role Immune Reconstitution Inflammatory Syndrome [IRIS] may play in increasing the frequency of periodontitis in HIV infected individuals under HAART. Though this proposed link is in its infantile stage, it merits due consideration keeping in view the increased prevalence of periodontitis in HIV infected individuals. Besides, periodontitis being definitely treatable and most often preventable, prevention and treatment of periodontitis in HIV infected patients could be 'a stitch in time' that could 'save nine. The present article is a compilation of initial evidence that suggests a probable role of periodontitis in HIV re-activation; an attempt to invite more attention to this potential link.

\section{Periodontitis and HIV Reactivation}

Immune and oral epithelial cells latently infected with HIV in gingival tissues may be an important source for HIV reactivation during chronic inflammatory events triggered by oral pathogens [3]. Evidence suggests that cytokines such as interleukin-1 [IL-1] and tumour necrosis factor- $\alpha$ [TNF- $\alpha$ ] activate nuclear factor kappa $B$ [NFkB], the principal transcription factor which can induce viral gene expression through interaction with the long terminal repeat [LTR] of the HIV provirus [4]. Interferon- $\gamma$ [IFN- $\gamma$ ], interleukin-6 [IL-6] and TNF- $\alpha$ upregulate IFN regulatory factor-1 [IRF-1] which leads to cell activation

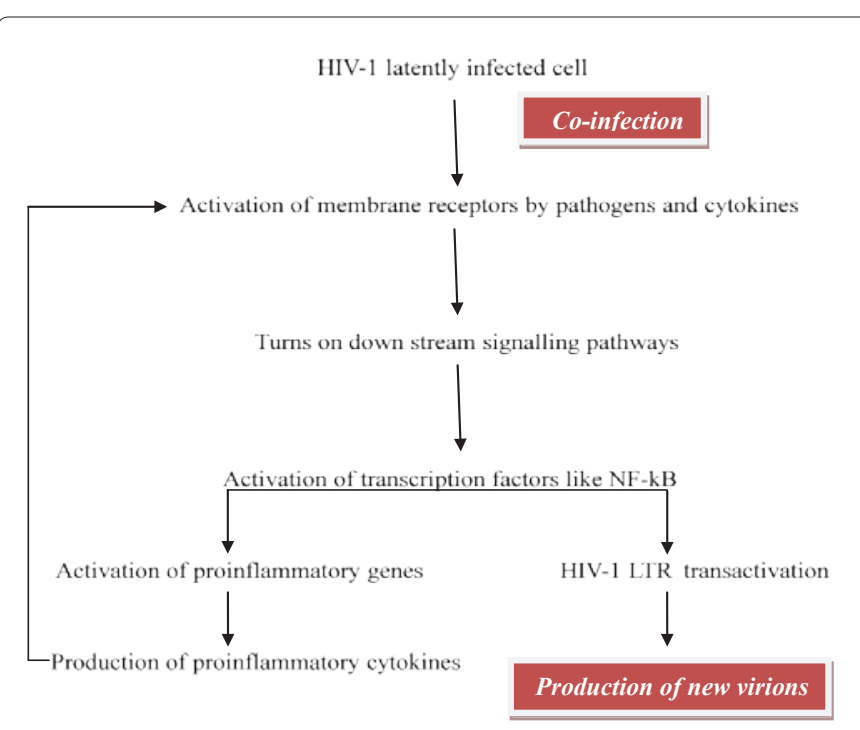

Figure 1 : Proposed Model Of HIV Reactivation During Co-Infection.

and drives HIV-1 replication [5]. Inflammatory mediator prostaglandin E2 also seems to be able to increase viral replication in T cells. IL-2 and IL-7 have also found to upregulate HIV activation in T cells [6]. Studies have shown that levels of all these inflammatory mediators are elevated in periodontitis suggesting a probable role of periodontitis in reactivation of latent HIV (Figure.1).

${ }^{*}$ Corresponding author: Ashita S Uppoor, MDS, Professor \& Head, Departmen of Periodontics, Manipal College of Dental Sciences, Mangalore, Karnataka, India, Tel: 0091 9880038082; E-mail: uash55@hotmail.com

Received August 11, 2012; Accepted September 17, 2012; Published Septembe 19, 2012

Citation: Uppoor AS, Nayak DG (2012) HIV and Periodontal Disease: Redemption or Resurrection. J AIDS Clinic Res 3:168. doi:10.4172/2155-6113.1000168

Copyright: ( 2012 Uppoor AS, et al. This is an open-access article distributed under the terms of the Creative Commons Attribution License, which permits un restricted use, distribution, and reproduction in any medium, provided the original author and source are credited. 
Imai et al. [7] demonstrated that Porphyromonas gingivalis produces high concentration of butyric acid, which is an inhibitor of histone deacetylases (HDAC). Hypoacetylation of histone proteins by HDAC is involved in the maintenance of HIV-1 latency by repressing viral transcription. Butyric acid being an inhibitor of HDAC strongly induces histone acetylation and activation of latent HIV-1 at the transcriptional level.

Gonzalez et al. [8] sought to determine the role of toll-like receptor 2 (TLR-2), TLR-4 and TLR-9 in HIV-1 reactivation in monocytes/ macrophages, induced by the periodontopathogens Fusobacterium nucleatum and $P$. gingivalis. They demonstrated that TLR-9 activation by $F$. nucleatum and TLR-2 activation by both the bacteria might be involved in HIV-1 reactivation.

Data from the study by Huang et al. [9] further support the hypothesis that oral bacteria related to periodontal infections could trigger latently infected dendritic cells in gingival tissues and contribute to HIV recrudescence. Moreover, it has demonstrated that $P$. gingivalis could up regulate CCR5, the principal HIV co-receptor, in oral keratinocytes and pave way to subsequent infection of immune cells in a CCR5 dependent manner [10]. In addition, studies have also shown correlation between clinical stage of periodontitis and HIV-1 proviral DNA load in gingival crevicular fluid as well as HIV-1 viral RNA load in plasma [11]. All these findings suggest that periodontal pathogens could contribute to reactivation of latent virus and progression of AIDS.

\section{Immune Reconstitution Inflammatory Syndrome and Haart}

Immune reconstitution is defined as a CD4+ count of $>200$ cells/ $\mathrm{mm}^{3}$ and an increase of $\geq 100$ cells over baseline any time since starting HAART [12]. Immune Reconstitution Inflammatory Syndrome (IRIS) caused by an overwhelming inflammatory response to a viable, dormant or nonviable antigen in patients with rapidly recovering immune system. IRIS could be 'unmasking' when a subclinical infection is newly identified after initiation of HAART or it could be 'paradoxical' in which clinical worsening of an existing condition occurs despite successful treatment [13].

Immunopathogenesis of IRIS is considered to be related to the recovery of $\mathrm{CD} 4+\mathrm{T}$ cells in response to antiretroviral therapy. The initial recovery is marked by an increase in circulating memory CD4+ T cells followed by a second phase in which there is recovery of naive activated $\mathrm{CD} 4+$ cells. The memory $\mathrm{T}$ cells giving rise to a vigorous antigen specific immune response could be responsible for the appearance of IRIS early in the recovery process [13]. Moreover, an interference with T-regulatory cell signalling is also implicated which leads to an exaggerated inflammatory response to the infecting antigen [14].

Data from various studies reveal varying results with respect to the frequency and type of oral lesions related to IRIS [15]. Oral lesions possibly related to IRIS include oral warts, erythematous and pseudomembranous candidiasis, angular chelitis, hairy leukoplakia and Kaposi's sarcoma $[15,16]$. IRIS may lead to increased frequency of periodontal disease as the presence of latent infection has been considered as a risk factor for the syndrome $[17,18]$. If IRIS results in exacerbation of subclinical periodontal infection in HIV patients, limited research findings available suggest that it may contribute to reactivation of latent HIV in immune and oral epithelial cells in the gingival tissue $[3,9]$.

\section{Conclusion}

Introduction of HAART has converted HIV infection into a 'chronic disease' but there is mounting evidence that co-infections and inflammation might result in reactivation of the latent virus and undermine the success of HAART. Periodontitis being prevalent among HIV infected individuals, its possible role in HIV reactivation merits further investigation. At present, there is lack of substantive evidence to support this hypothesis and extensive research would have to be undertaken before we can arrive at a conclusion.

\section{References}

1. (1993) Classification and diagnostic criteria for oral lesions in HIV infection. EC-Clearinghouse on Oral Problems Related to HIV Infection and WHO Collaborating Centre on Oral Manifestations of the Immunodeficiency Virus. J Oral Pathol Med 22: 289-291.

2. Coogan MM, Greenspan J, Challacombe SJ (2005) Oral lesions in infection with human immunodeficiency virus. Bull World Health Organ 83: 700-706.

3. González OA, Ebersole JL, Huang CB (2009) Oral infectious diseases: A potential risk factor for HIV virus recrudescence? Oral Dis 15: 313-327.

4. Folks TM, Clouse KA, Justement J, Rabson A, Duh E, et al. (1989) Tumor necrosis factor alpha induces expression of human immunodeficiency virus in a chronically infected T-cell clone. Proc Natl Acad Sci USA 86: 2365-2368.

5. Fauci AS (1996). Host factors and the pathogenesis of HIV-induced disease Nature 384: 529-534.

6. Wang FX, Xu Y, Sullivan J, Souder E, Argyris EG, et al. (2005) IL-7 is a potent and proviral strain-specific inducer of latent HIV-1 cellular reservoirs of infected individuals on virally suppressive HAART. J Clin Invest 115: 128-137.

7. Imai K, Ochiai K, Okamoto T (2009) Reactivation of latent HIV-1 infection by periodontopathic bacterium Porphyromonas gingivalis involves histone modification. J Immunol 182: 3688-3695.

8. González OA, Li M, Ebersole JL, Huang CB (2010) HIV-1 reactivation induced by the periodontal pathogens Fusobacterium nucleatum and Porphyromonas gingivalis involves toll-like receptor 2 [corrected] and 9 activation in monocytes/ macrophages. Clin Vaccine Immunol 17: 1417-1427.

9. Huang CB, Alimova YV, Strange S, Ebersole JL (2011) Polybacterial challenge enhances HIV reactivation in latently infected macrophages and dendritic cells. Immunology 132: 401-409.

10. Giacaman RA, Asrani AC, Gebhard KH, Dietrich EA, Vacharaksa A, et al. (2008) Porphyromonas gingivalis induces CCR5-dependent transfer of infectious HIV1 from oral keratinocytes to permissive cells.Retrovirology 5: 29 .

11. Maticic M, Poljak M, Kramar B, Tomazic J, Vidmarl L, et al. (2000) Proviral HIV1 DNA in gingival crevicular fluid of HIV-1-infected patients in various stages of HIV disease. J Dent Res 79: 1496-1501.

12. Arici C, Ripamonti D, Ravasio V, Maggiolo F, Rizzi M, et al. (2001) Long-term clinical benefit after highly active antiretroviral therapy in advanced HIV-1 infection, even in patients without immune reconstitution. Int J STD AIDS 12 $573-581$.

13. Tappuni AR (2011) Immune reconstitution inflammatory syndrome. Adv Dent Res 23: 90-96.

14. Kestens L, Seddiki N, Bohjanen PR (2008) Immunopathogenesis of immune reconstitution disease in HIV patients responding to antiretroviral therapy. Cur Opin HIV AIDS 3: 419-424.

15. Gaitan Cepeda LA, Ceballos Salobreña A, López Ortega K, Arzate Mora $\mathrm{N}$, Jiménez Soriano $\mathrm{Y}$ (2008) Oral lesions and immune reconstitution syndrome in HIV+/AIDS patients receiving highly active antiretroviral therapy. Epidemiological evidence. Med Oral Patol Oral Cir Bucal 13: E85-93.

16. Patton LL, McKaig R, Strauss R, Rogers D, Eron JJ Jr (2000) Changing prevalence of oral manifestations of human immuno-deficiency virus in the era of protease inhibitor therapy. Oral Surg Oral Med Oral Pathol Oral Radiol Endod 89: $299-304$

17. Crum-Cianflone NF (2006) Immune reconstitution inflammatory syndromes: what's new? AIDS Read 16:199-206.

18. Murdoch DM, Venter WD, Van Rie A, Feldman C (2007) Immune reconstitution inflammatory syndrome (IRIS): Review of common infectious manifestations and treatment options. AIDS Res Ther 4: 9. 